\title{
Nanoindentation and Adhesion Properties of Ta Thin Films
}

\author{
Yuan-Tsung Chen \\ Department of Materials Science and Engineering, I-Shou University, Kaohsiung 840, Taiwan \\ Correspondence should be addressed to Yuan-Tsung Chen; ytchen@isu.edu.tw
}

Received 1 February 2013; Revised 6 March 2013; Accepted 14 March 2013

Academic Editor: Ugur Serincan

Copyright (C) 2013 Yuan-Tsung Chen. This is an open access article distributed under the Creative Commons Attribution License, which permits unrestricted use, distribution, and reproduction in any medium, provided the original work is properly cited.

\begin{abstract}
Ta films were sputtered onto a glass substrate with thicknesses from $500 \AA$ to $1500 \AA$ under the following conditions: (a) as-deposited films were maintained at room temperature (RT), (b) films were postannealed at $T_{A}=150^{\circ} \mathrm{C}$ for $1 \mathrm{~h}$, and (c) films were postannealed at $T_{A}=250^{\circ} \mathrm{C}$ for $1 \mathrm{~h}$. X-ray diffraction (XRD) results revealed that the Ta films had a body-centered cubic (BCC) structure. Postannealing conditions and thicker Ta films exhibited a stronger Ta (110) crystallization than as-deposited and thinner films. The nanoindention results revealed that Ta thin films are sensitive to mean grain size, including a valuable hardness $(H)$ and Young's modulus $(E)$. High nanomechanical properties of as-deposited and thinner films can be investigated by grain refinement, which is consistent with the Hall-Petch effect. The surface energy of as-deposited Ta films was higher than that in postannealing treatments. The adhesion of as-deposited Ta films was stronger than postannealing treatments because of crystalline degree effect. The maximal $H$ and $E$ and the optimal adhesion of an as-deposited 500 - $\AA$-thick Ta film were $15.6 \mathrm{GPa}, 180 \mathrm{GPa}$, and $51.56 \mathrm{~mJ} / \mathrm{mm}^{2}$, respectively, suggesting that a 500 - $\AA$-thick Ta thin film can be used in seed and protective layer applications.
\end{abstract}

\section{Introduction}

The study of Ta performance is noteworthy because it is a crucial material. Ta is generally inserted as a seed layer, buffer layer, capping layer, and protective layer in a multilayered structure, which can be used extensively in magnetoresistance random access memory (MRAM) and semiconductor applications [1-5]. It is compatible with the semiconductor process and can also be used as a predeposited layer on substrate or combined with other layers in the epitaxial system, such as $\mathrm{Ta} / \mathrm{CoCrPt}, \mathrm{Ta} / \mathrm{Co}$, and $\mathrm{Ta} / \mathrm{SiO}_{2} / \mathrm{Si}$ systems [6-8]. The superior Ta seed layer needs to have high mechanical properties and strong adhesion, including high hardness $(H)$, Young's modulus $(E)$, and surface energy because Ta seed layer is usually deposited and combined with other layers. Moreover, the performance of Ta-seed multilayered structure is sensitive to a temperate environment. However, few studies have focused on the hardness, Young's modulus, contact angle, surface energy, and adhesion properties of Ta thin films at room temperature (RT) and postannealing temperature conditions. These few studies only investigated about magnetic and electrical properties of Ta seed multilayered structure [2-4]. In order to justify the contribution of the presented study, the nanomechanical properties and adhesion of Ta thin films in as-deposited and postannealing conditions are worth investigating in detail.

An experiment was conducted to determine the structure, nanomechanical properties, and adhesion of Ta films with $\mathrm{X}$-ray diffraction (XRD), nanoindentation measurement, and contact angle results. The nanoindentation observation yielded considerable information on mechanical properties (hardness and elastic modulus), based on analyses of loaddisplacement curves [9-12]. The results indicate that the high nanomechanical properties of as-deposited and thinner films can be investigated by grain refinement, which is consistent with the Hall-Petch effect. Surface energy was calculated by contact average angle to understand the adhesion of Ta thin films. The surface energy of as-deposited Ta films was higher than that in postannealing treatments. This indicates that adhesion of as-deposited Ta films was stronger than postannealing treatments because of crystalline degree effect. 


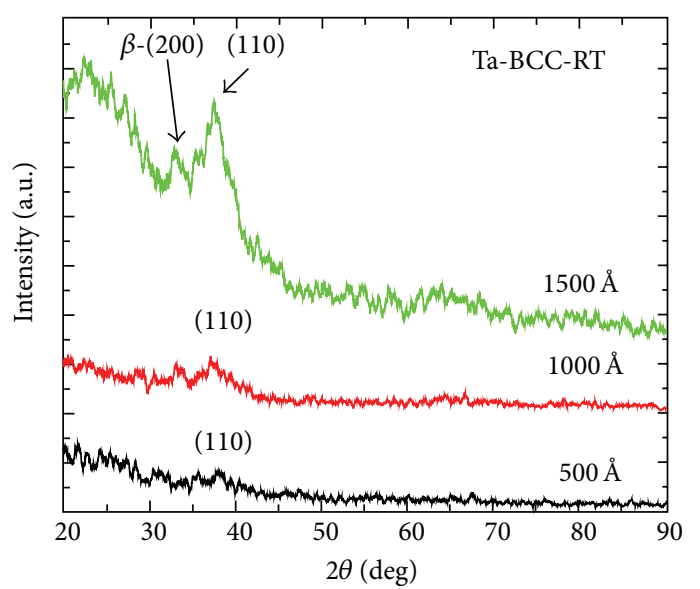

(a)

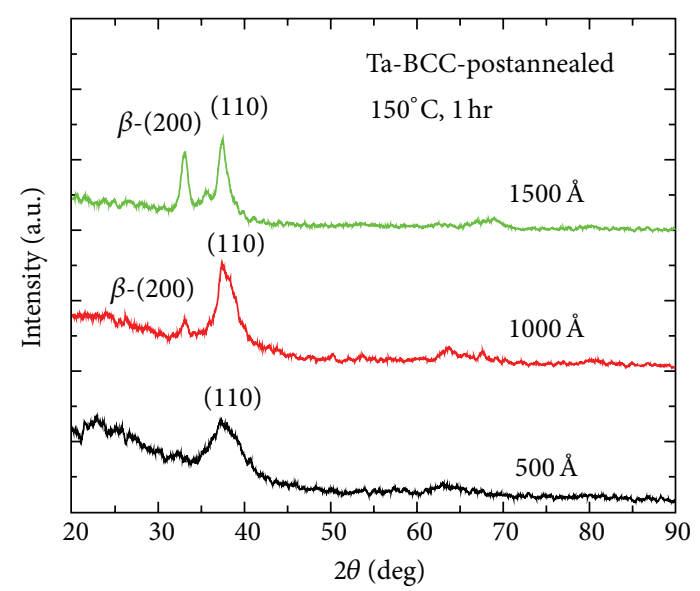

(b)

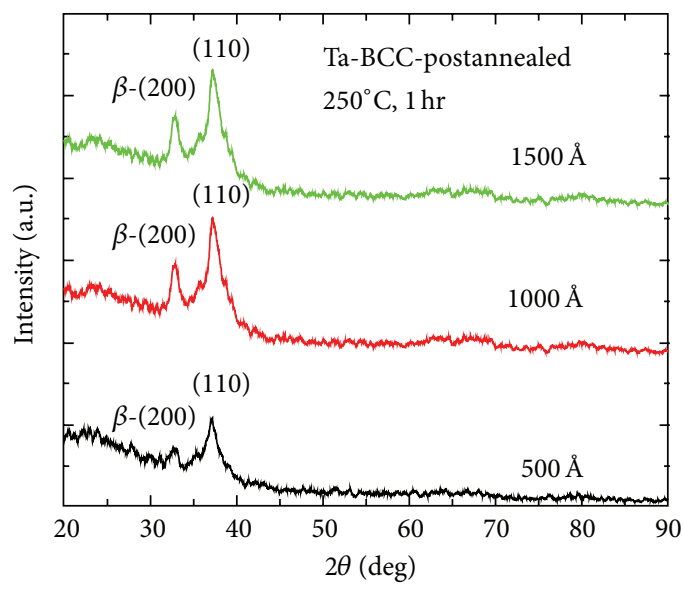

(c)

Figure 1: XRD results for Ta films at three conditions. (a) Deposited at RT only, (b) postannealing at $T_{A}=150^{\circ} \mathrm{C}$ for $1 \mathrm{~h}$, and (c) postannealing at $T_{A}=250^{\circ} \mathrm{C}$ for $1 \mathrm{~h}$.

\section{Experimental}

Ta thin films were deposited onto a glass substrate by $\mathrm{dc}$ magnetron sputtering, with thicknesses $\left(t_{f}\right)$ from $500 \AA$ to $1500 \AA$, under the following three conditions: (a) substrate temperature $\left(T_{s}\right)$ maintained at RT; (b) $T_{s}=\mathrm{RT}$, followed by postannealing at $T_{A}=150^{\circ} \mathrm{C}$ for $1 \mathrm{~h}$; (c) postannealing at $T_{A}=250^{\circ} \mathrm{C}$ for $1 \mathrm{~h}$. The postannealing conditions are under inert gas Argon (Ar) atmosphere at chamber pressure $2 \times$ $10^{-3}$ Torr. This procedure can solve the oxidation problem of Ta surface [13]. The typical base chamber pressure exceeded $1.5 \times 10^{-7}$ Torr, and the Ar working chamber pressure was $5 \times 10^{-3}$ Torr. The structure of the Ta thin film was characterized by using XRD with a $\mathrm{CuK}_{\alpha 1}$ line (Philips X'Pert). The average grain size was estimated by full width at half maximum (FWHM, B) and Scherrer's formula. The hardness and Young's modulus of the Ta thin films were measured using an MTS Nanoindenter XP with a Berkovich indenter. Ta thin films can be used intensively in MRAM and/or semiconductor applications. In MRAM applications, thin Ta layers with thicknesses below $100 \AA$ are used as adhesion, such as a seed and/or cap (protection) layer. In the present study, Ta films with thicknesses in the range of $500 \AA$ to $1500 \AA$ were investigated. In order to investigate nanomechanical results of Ta thin films at nanoindention measured process, the thicker Ta thickness is suitable than thinner thickness to avoid substrate effect. An indentation load of $1 \mathrm{mN}$ was used to limit the depth of penetration of the indenter to less than $10 \%$ of the film thickness. The indentation load was increased in 40 steps, and the penetration depth was measured at each step. Six indentations were investigated in each sample and averaged for more accurate results with a standard deviation. Moreover, the surface energy of the Ta films was calculated from measurement of the contact angle, with water and diiodomethane as test liquids. The surface energy is obtained from the contact angle and some calculations [14-16].

\section{Results and Discussion}

Figure 1 shows the X-ray diffraction patterns of Ta films under three conditions. Figures $1(\mathrm{a})-1(\mathrm{c})$ show the X-ray results for the films formed at RT, with postannealing at 


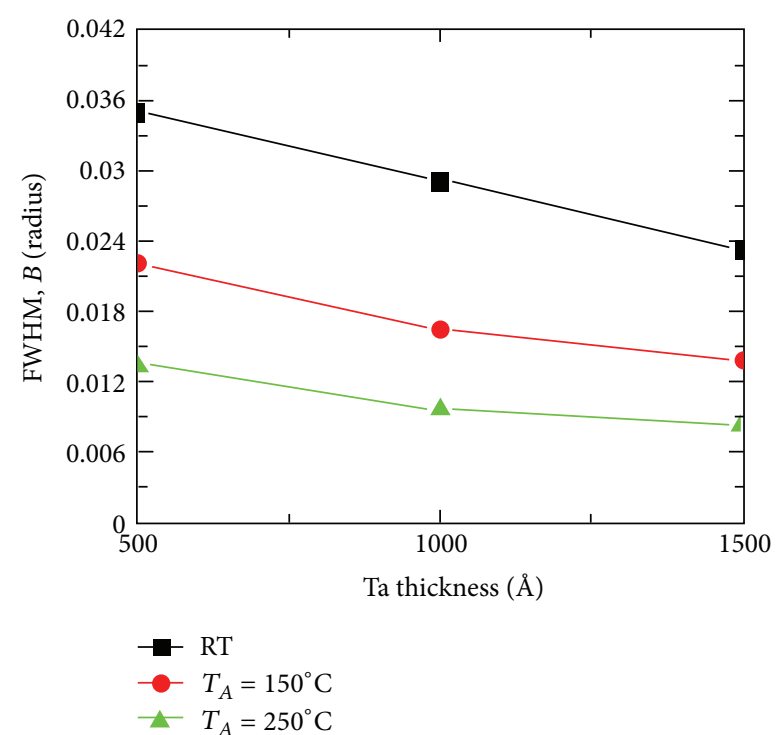

FIgURE 2: FWHM (B) of (110) peak as a function of thickness of Ta thin films prepared under three conditions.

$T_{A}=150^{\circ} \mathrm{C}$ and $T_{A}=250^{\circ} \mathrm{C}$. XRD results indicated that the Ta thin films have a body-centered cubic (BCC) structure. This result indicates that Ta films include the substantial crystalline peaks, with a highly crystalline (110) diffraction peak and $\beta$-(200) peak at $1000 \AA$ and $1500 \AA$, respectively, under postannealing conditions. However, only main weak (110) peak of Ta $500 \AA$ was found at RT and postannealing condition of $150^{\circ} \mathrm{C}$. The main (110) peaks with the weak $\beta$ (200) peak were found at postannealing conditions of $250^{\circ} \mathrm{C}$ at $500 \AA$. Figure 1(a) shows that the as-deposited Ta film has a weak crystallization of $\mathrm{Ta}$ (110) diffraction peak. Figures 1(b)1 (c) show a multigrain crystalline phenomenon, including (110) and $\beta$-(200) diffraction peaks. In addition, the thicker thickness and postannealing treatment shown in Figures 1(b)-1(c) can induce stronger diffracted intensity than thinner thickness and as-deposited treatment. The diffraction peaks of the Ta thin films became more crystalline as the thickness increased. Crystallization achieved by annealing treatment exceeded that achieved at RT because thermal energy drives grain growth.

The corresponding FWHM (B) of the main Ta (110) peak at three different thicknesses under three conditions is shown in Figure 2. Scherrer's relation was used to estimate mean grain size because the Ta (110) of XRD diffraction result indicated that the intensity of the peak was larger than $\beta$-Ta (200) [17]. Figure 2 shows that weak crystallization occurred under the as-deposited condition and thinner Ta film because it yields a larger FWHM. In contrast, strong crystallization occurred in the postannealing condition and thicker Ta film because of a smaller FWHM. The FWHM for films formed at RT and thin thickness was larger than that of films that have undergone postannealing and thick thickness.

The mean crystalline grain size $(D)$ can be obtained from the measured width of the diffraction peak under crystalline

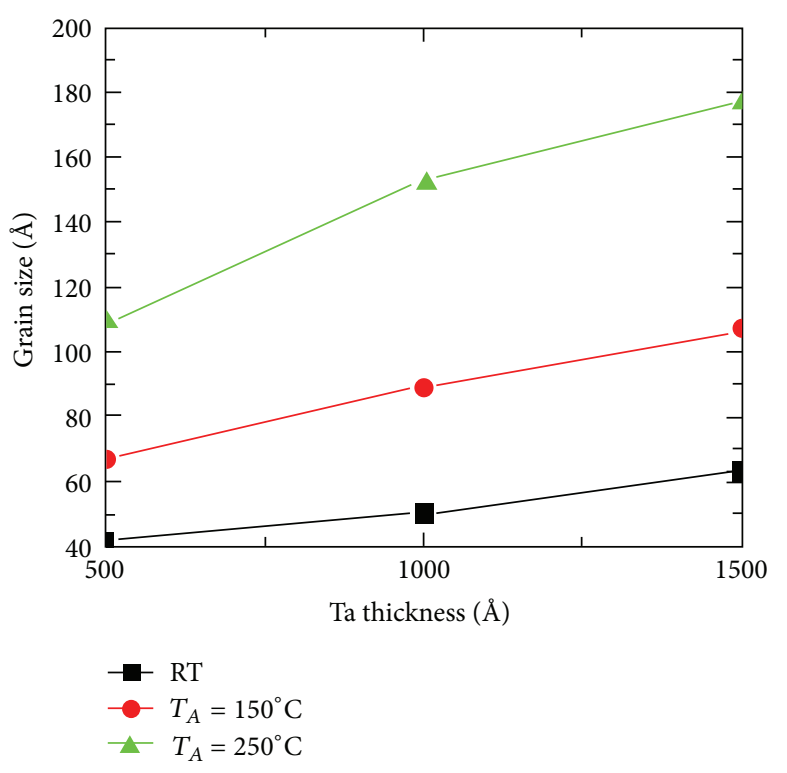

FIgURE 3: Mean grain size as a function of thickness of Ta thin films prepared under three conditions.

quality of three conditions, using Scherrer's formula for estimation, as shown in Figure 3. Scherrer's formula is listed [17].

Consider the following:

$$
D=\frac{k \lambda}{B \cos \theta},
$$

where $k(0.9)$ is Scherrer's constant, $\lambda$ is the $\mathrm{X}$-ray wavelength of the $\mathrm{CuK}_{\alpha 1}$ line, $B$ is the relative value of the FWHM of the (110) peak, and $\theta$ is the half angle of the diffraction peak. The formula states that $D$ is proportional to $1 / B$; thus, a larger $B$ corresponds to smaller grains. Scherrer's formula yielded mean grain sizes of $63 \AA, 107 \AA$, and $178 \AA$ for 1500 - $\AA$-thick Ta thin films under the following conditions: (a) with a substrate temperature $\left(T_{s}\right)$ maintained at RT; (b) after postannealing at $T_{A}=150^{\circ} \mathrm{C}$ for $1 \mathrm{~h} ;$ (c) after postannealing and heat annealing at $T_{A}=250^{\circ} \mathrm{C}$ for $1 \mathrm{~h}$.

Figure 4(a) shows the hardness $(H)$ of Ta samples under three conditions. Higher values of Ta films were observed in as-deposited and thin Ta films because the grain-refined effect induced the "Hall-Petch" phenomenon. Furthermore, the same result of Young's modulus $(E)$ of Ta films is shown in Figure 4(b). Similarly, the larger values of Ta films occurred in as-deposited and thin Ta films. It also suggests that the "Hall-Petch" equation is suitable for fitting in the Ta layer sufficiently. This indicates that the smaller grains can enhance higher $H$ and $E$ values than larger grains, which also explains a strong nanomechanical performance at asdeposited and thinner films. The result of Ta thin films is consistent with the CoFeB and NiFe thin films [18-20]. The $H$ and $E$ of Ta films declined as the thickness increased, and the postannealing temperature was enhanced. Figure 4 shows that the highest $H$ and $E$ of the Ta films were $15.6 \mathrm{GPa}$ and 


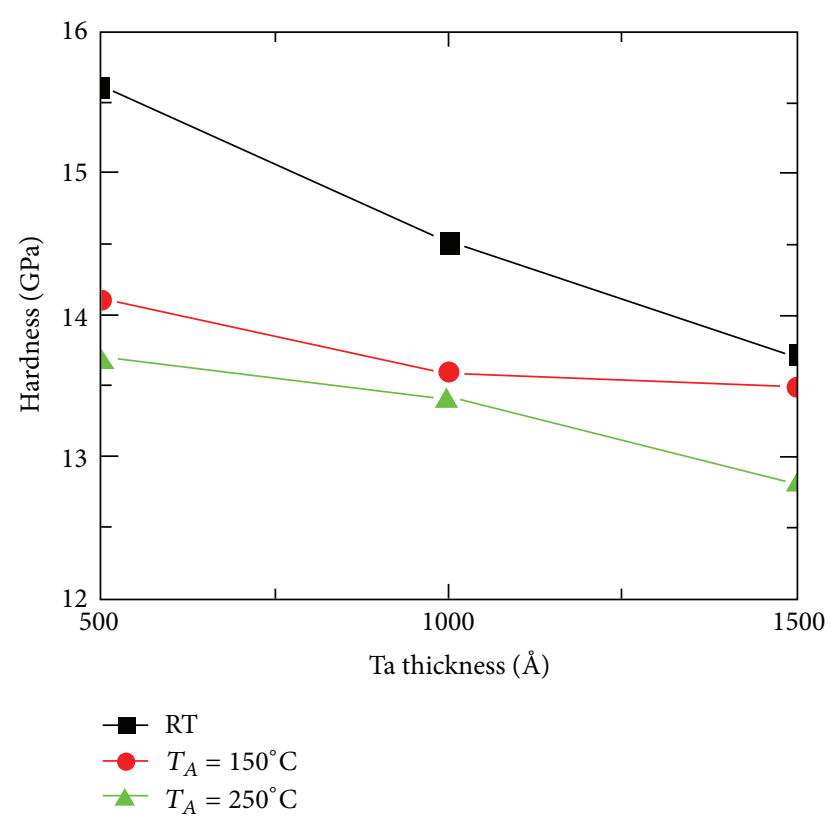

(a)

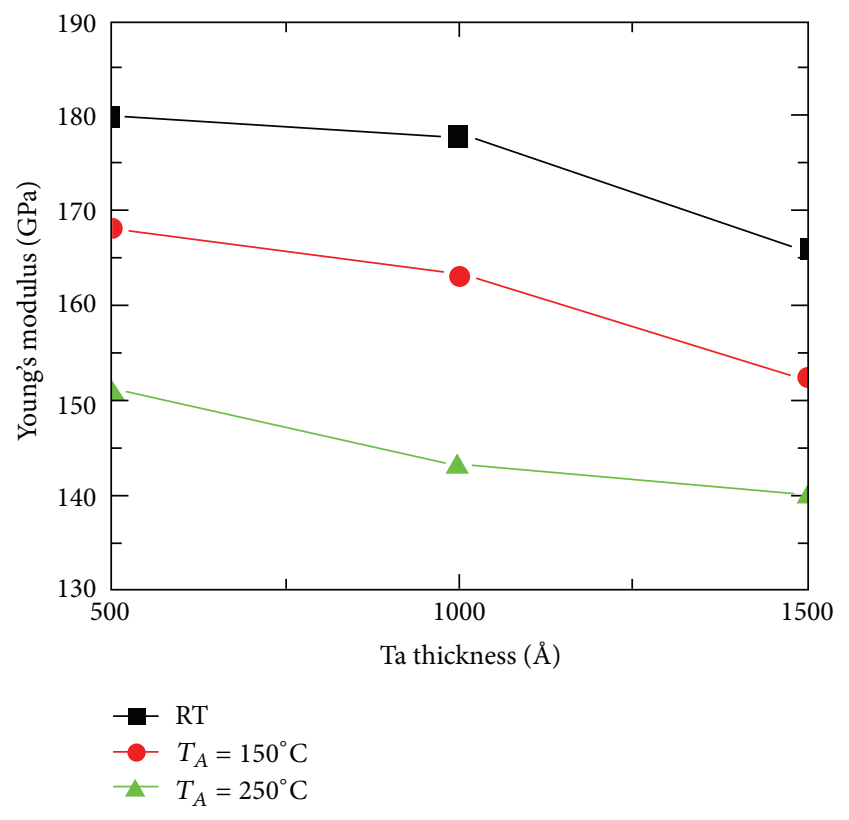

(b)

FIgURE 4: Nano-mechanical properties of Ta samples under three conditions. (a) Hardness and (b) Young's modulus.

$180 \mathrm{GPa}$, respectively, at $500 \AA$ of the as-deposited Ta samples, which can be adjusted for seed layer and capping layer applications.

Hardness levels are known to be directly related to grain size, as demonstrated by the Hall-Petch equation [21]:

$$
H=H_{i}+k D^{-1 / 2} \text {. }
$$

$H_{i}$ represents lattice friction stress, $k$ represents the HallPetch constant, and $D$ represents the mean grain size.
This formula demonstrates that finer grains are associated with more grain boundaries and induce greater mechanical strength. Figures $5(\mathrm{a})-5(\mathrm{c})$ show that the dashed line is a linear equation fitting plot at three conditions. The Hall-Petch equation was used with linear fitting to obtain the result in Figure 5(a)

$$
H=5.5+64.9 D^{-1 / 2} \text {. }
$$

This equation uses a probable lattice friction stress of $5.5 \mathrm{GPa}$ and a Hall-Petch constant of $64.9 \mathrm{GPa} \AA^{-1 / 2}$ for the asdeposited Ta thin films, which is shown in Figure 5(a). The Hall-Petch equation was also used with linear fitting to reach the result in Figure 5(b)

$$
H=10.2+33.5 D^{-1 / 2}
$$

This equation uses a probable lattice friction stress of $10.2 \mathrm{GPa}$ and a Hall-Petch constant of $33.5 \mathrm{GPa} \AA^{-1 / 2}$ for the postannealed $150^{\circ} \mathrm{C}$ Ta thin films. Moreover, the Hall-Petch equation was also used with linear fitting to reach the result in Figure 5(c)

$$
H=9.9+39.7 D^{-1 / 2} \text {. }
$$

This equation uses a probable lattice friction stress of $9.9 \mathrm{GPa}$ and a Hall-Petch constant of $39.7 \mathrm{GPa} \AA^{-1 / 2}$ for the postannealed $250^{\circ} \mathrm{C}$ Ta thin films. About the linear fitting discrepancy in Figures 5(b)-5(c), it can be reasonably concluded that the Berkovich indenter of MTS Nanoindenter XP at nanoindention measured process touches some defects or impurity and induces a linear fitting discrepancy. The slope of the Hall-Petch equation at RT is larger than the Hall-Petch equation using postannealing treatments, which indicates that the refined grains at RT sufficiently and efficiently affect the hardness levels. This result is consistent with the "HallPetch" effect.

Figures 6(a)-6(b) show the contact angle results of Ta films under three conditions with water and diiodomethane as the test liquids. The contact angles of all films were smaller than $90^{\circ}$, which indicates that the Ta films are hydrophilic. The trend of the contact angle shows that the as-deposited thinner films were smaller than the postannealed thicker films with water as the test liquid. In contrast, the trend of the contact angle shows that the as-deposited thinner films were larger than postannealed thicker films with diiodomethane as the test liquid. Therefore, the various thicknesses, asdeposited condition, and postannealing treatments were sensitive to the contact angle because of crystalline degree of films [22]. The corresponding surface energy of Ta films was obtained by contact angle calculation, as shown in Figure 6(c) [14-16]. This figure shows that the surface energy of asdeposited thinner films was larger than that of postannealed thicker films. It suggests that the weaker crystallization and smaller mean grain size caused a high surface energy and strong adhesion. The surface energy is a crucial index to estimate the adhesion of films. The higher surface energy 

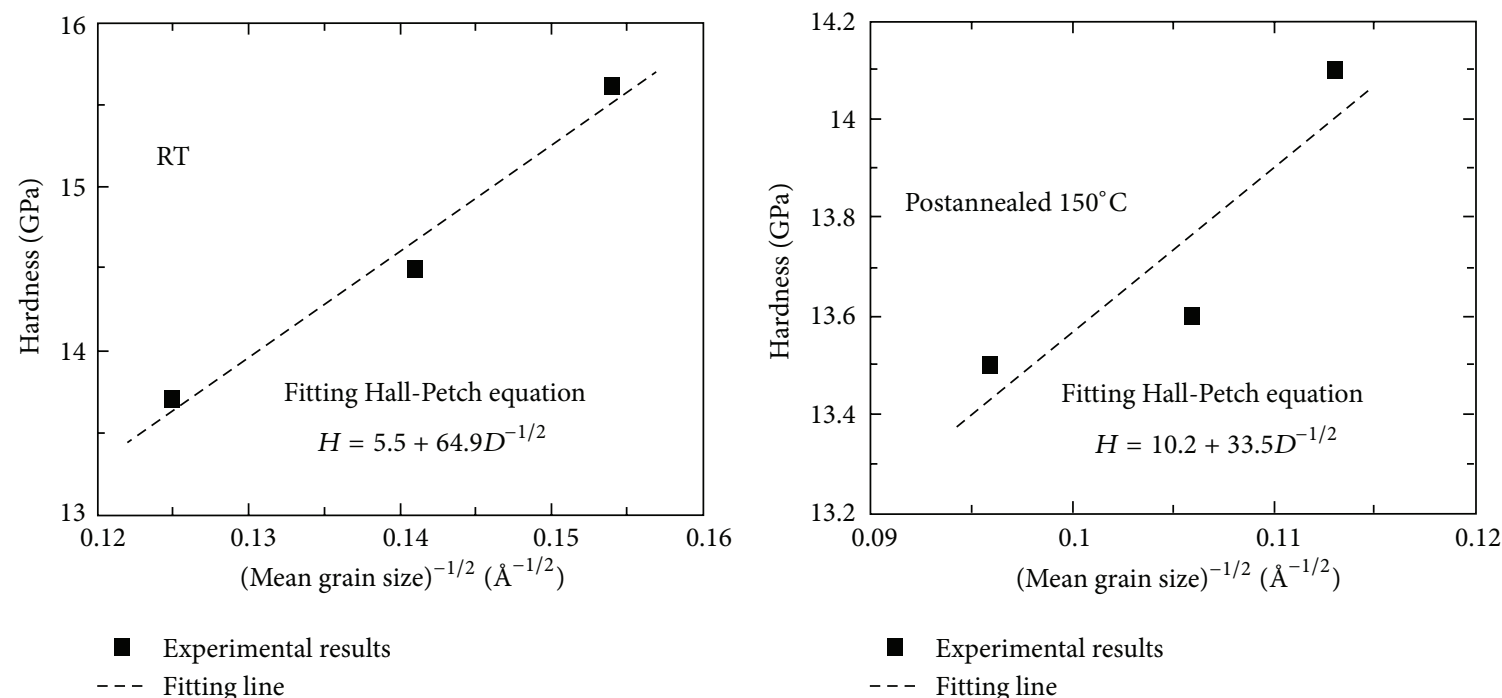

(a)

(b)

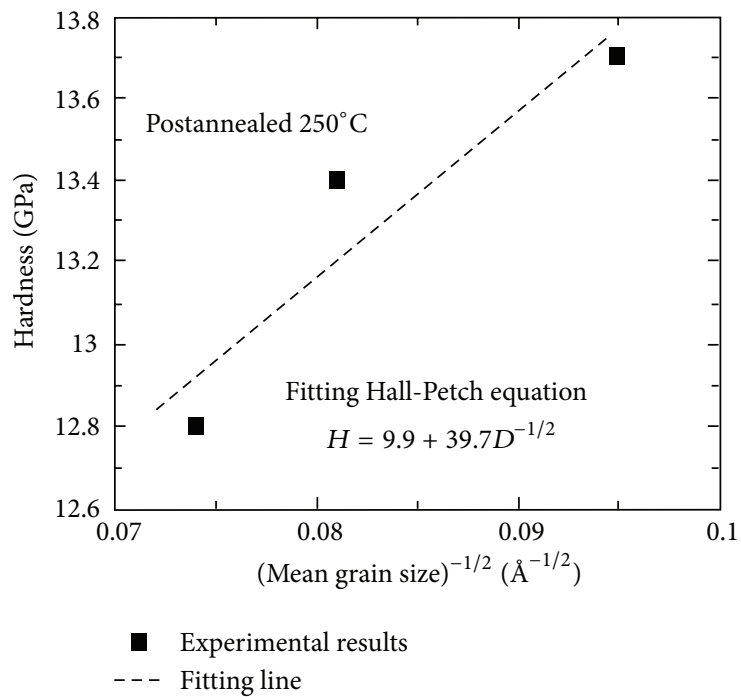

(c)

FIGURE 5: Experimental hardness as a function of mean grain size, fitted using the Hall-Petch equation. (a) Was deposited at RT only, (b) postannealed at $150^{\circ} \mathrm{C}$ for $1 \mathrm{~h}$, and (c) postannealed at $250^{\circ} \mathrm{C}$ for $1 \mathrm{~h}$.

indicated that the film had a stronger adhesion. Based on this result, the optimal surface energy was $51.56 \mathrm{~mJ} / \mathrm{mm}^{2}$ at $500 \AA$ of the as-deposited Ta sample, which is suitable for use as a seed layer and buffer layer in MRAM application and semiconductor process.

\section{Conclusion}

In conclusion, the experiment revealed the nanomechanical and adhesive properties of as-deposited and postannealed Ta thin films of various thicknesses under three conditions. The XRD structure and mean grain size show that strong crystallization and a large mean grain size occurred under the postannealing condition and thicker thickness. Moreover, the high nanomechanical phenomenon of Ta films was observed at the as-deposited condition and thin thickness, which matched the "Hall-Petch" effect, and was suitable for use in seed layer and capping layer applications because of grain refinement. The highest surface energy and adhesion of Ta films were obtained at $500 \AA$ of as-deposited Ta film. Based on the nanomechanical and adhesive properties, the optimal Ta condition was observed at $500 \AA$ of the as-deposited Ta film, and the highest $H, E$, and surface energy were $15.6 \mathrm{GPa}, 180 \mathrm{GPa}$, and $51.56 \mathrm{~mJ} / \mathrm{mm}^{2}$, respectively. Therefore, it is suitable for combination with other layers or to protect other films in magnetic field and semiconductor process applications. 

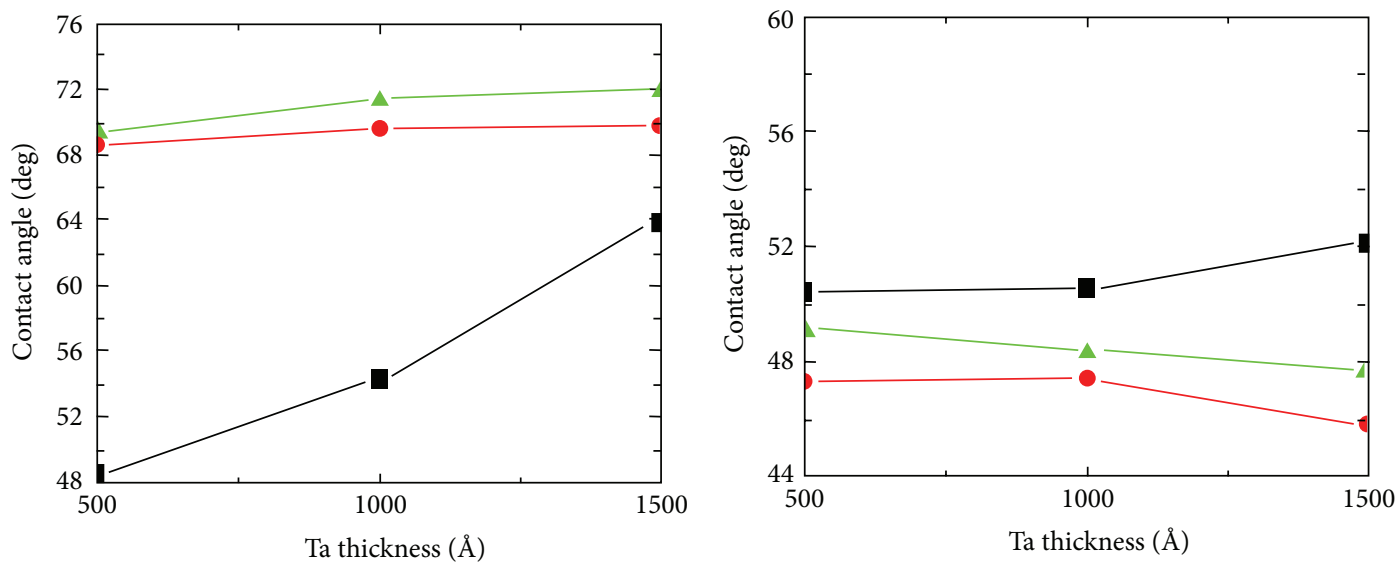

By test liquid water

By test liquid diiodomethane

$$
\begin{aligned}
& \longrightarrow \mathrm{RT} \\
& -T_{A}=150^{\circ} \mathrm{C} \\
& -T_{A}=250^{\circ} \mathrm{C}
\end{aligned}
$$

- $\mathrm{RT}$

- $T_{A}=150^{\circ} \mathrm{C}$

$-T_{A}=250^{\circ} \mathrm{C}$

(a)

(b)

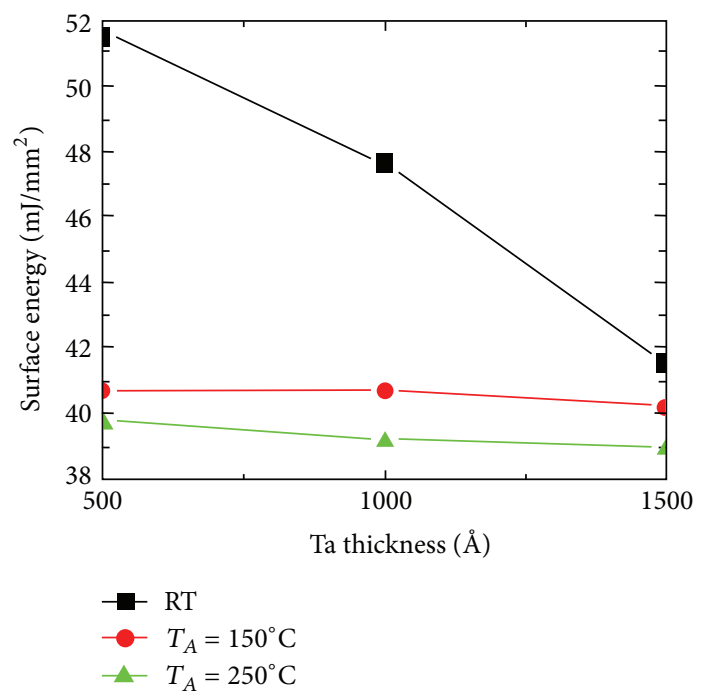

(c)

Figure 6: Adhesion properties of Ta samples under three conditions. (a) Contact angle with test liquid water, (b) contact angle with test liquid diiodomethane, and (c) surface energy.

\section{Acknowledgment}

This work was supported by the National Science Council, under Grant no. NSC100-2112-M214-001-MY3. The author would like to thank for experimental assistance of Professor C. F. Wang.

\section{References}

[1] R. Law, R. Sbiaa, T. Liew, and T. C. Chong, "Effects of Ta seed layer and annealing on magnetoresistance in CoFe/Pd-based pseudo-spin-valves with perpendicular anisotropy," Applied Physics Letters, vol. 91, no. 24, Article ID 242504, 3 pages, 2007.
[2] M. Kowalewski, W. H. Butler, N. Moghadam et al., "The effect of Ta on the magnetic thickness of permalloy $\left(\mathrm{Ni}_{81} \mathrm{Fe}_{19}\right)$ films," Journal of Applied Physics, vol. 87, no. 9, pp. 5732-5734, 2000.

[3] W. Peng, O. Keitel, R. H. Victora, E. Koparal, and J. H. Judy, "Co/Pt superlattices with ultra-thin $\mathrm{Ta}$ seed layer on $\mathrm{NiFe}$ underlayer for double-layer perpendicular magnetic recording media," IEEE Transactions on Magnetics, vol. 36, no. 5 I, pp. 2390-2392, 2000.

[4] K. Weiss, S. Riedel, S. E. Schulz et al., "Development of different copper seed layers with respect to the copper electroplating process," Microelectronic Engineering, vol. 50, no. 1-4, pp. 433440, 2000.

[5] W. P. Jayasekara, S. Zhang, D. Mauri, S. Nguyen, and T. Shatz, "Etching of spin-valve capping layers for sensor stabilization 
applications," IEEE Transactions on Magnetics, vol. 39, no. 5, pp. 2381-2383, 2003.

[6] A. G. Roy and D. E. Laughlin, "Effect of seed layers in improving the crystallographic texture of CoCrPt perpendicular recording media," Journal of Applied Physics, vol. 91, no. 10, pp. 8076-8078, 2002.

[7] Y. T. Chen, Y. C. Lin, S. U. Jen, J. Y. Tseng, and Y. D. Yao, "Effect of Ta seed layer on crystalline structure and magnetic properties in an exchange-biased Co/IrMn system," Journal of Alloys and Compounds, vol. 509, no. 18, pp. 5587-5590, 2011.

[8] T. H. Meen, W. R. Chen, C. J. Huang, and C. J. Chiu, “Thin copper seed layers in interconnect metallization using the electroless plating process," Japanese Journal of Applied Physics, vol. 43, no. 8, pp. 5100-5104, 2004.

[9] S. U. Jen, Y. T. Chen, N. T. Yang, and W. C. Cheng, "Mechanical and structural properties of Permalloy films on glass and/or Si(111) substrates," Applied Physics A, vol. 94, no. 2, pp. 431-436, 2008.

[10] S. R. Jian, J. S. C. Jang, Y. S. Lai et al., "Mechanical properties of In GaN thin films deposited by metal-organic chemical vapor deposition," Materials Chemistry and Physics, vol. 109, no. 2-3, pp. 360-364, 2008.

[11] S. R. Jian, I. J. Teng, P. F. Yang et al., "Surface morphological and nanomechanical properties of PLD-derived $\mathrm{ZnO}$ thin films," Nanoscale Research Letters, vol. 3, no. 5, pp. 186-193, 2008.

[12] S. R. Jian, J. S. C. Jang, G. J. Chen, H. G. Chen, and Y. T. Chen, "Nanoindentation on a-plane $\mathrm{ZnO}$ thin films," Journal of Alloys and Compounds, vol. 479, no. 1-2, pp. 348-351, 2009.

[13] K. M. Yin, L. Chang, F. R. Chen et al., "Oxidation of Ta diffusion barrier layer for $\mathrm{Cu}$ metallization in thermal annealing," Thin Solid Films, vol. 388, no. 1-2, pp. 27-33, 2001.

[14] K. Ma, T. S. Chung, and R. J. Good, "Surface energy of thermotropic liquid crystalline polyesters and polyesteramide," Journal of Polymer Science B, vol. 36, no. 13, pp. 2327-2337, 1998.

[15] D. K. Owens and R. C. Wendt, "Estimation of the surface free energy of polymers," Journal of Applied Polymer Science, vol. 13, no. 8, pp. 1741-1747, 1969.

[16] D. H. Kaelble and K. C. Uy, "Reinterpretation of organic liquid-polytetrafluoroethylene surface interactions," Journal of Adhesion, vol. 2, pp. 50-60, 1970.

[17] B. D. Cullity, Elements of X-Ray Diffraction, Addison-Wesley, Reading, Mass, USA, 2nd edition, 1978.

[18] Y. T. Chen and C. C. Chang, "Effect of grain size on magnetic and nanomechanical properties of $\mathrm{Co}_{60} \mathrm{Fe}_{20} \mathrm{~B}_{20}$ thin films," Journal of Alloys and Compounds, vol. 498, no. 2, pp. 113-117, 2010.

[19] Y. T. Chen and C. W. Wu, "Effect of grain size on nanoindentation property of $\mathrm{Co}_{60} \mathrm{Fe}_{20} \mathrm{~B}_{20}$ thin film," Journal of Alloys and Compounds, vol. 551, pp. 360-364, 2013.

[20] Y. T. Chen and C. W. Wu, "Effect of grain size on nanomechanical property $\mathrm{Ni}_{80} \mathrm{Fe}_{20}$ thin film," Intermatallics, vol. 34, pp. 8993, 2013.

[21] R. Venkatraman and J. C. Bravman, "Separation of film thickness and grain boundary strengthening effects in Al thin films on Si," Journal of Materials Research, vol. 7, no. 8, pp. 2040-2048, 1992.

[22] S. K. Wang, Y. T. Chen, and S. R. Jian, "Determining contact angle and surface energy of $\mathrm{Co}_{60} \mathrm{Fe}_{20} \mathrm{~B}_{20}$ thin films by magnetron sputtering," Journal of Nanomaterials, vol. 2011, Article ID 291935, 4 pages, 2011. 

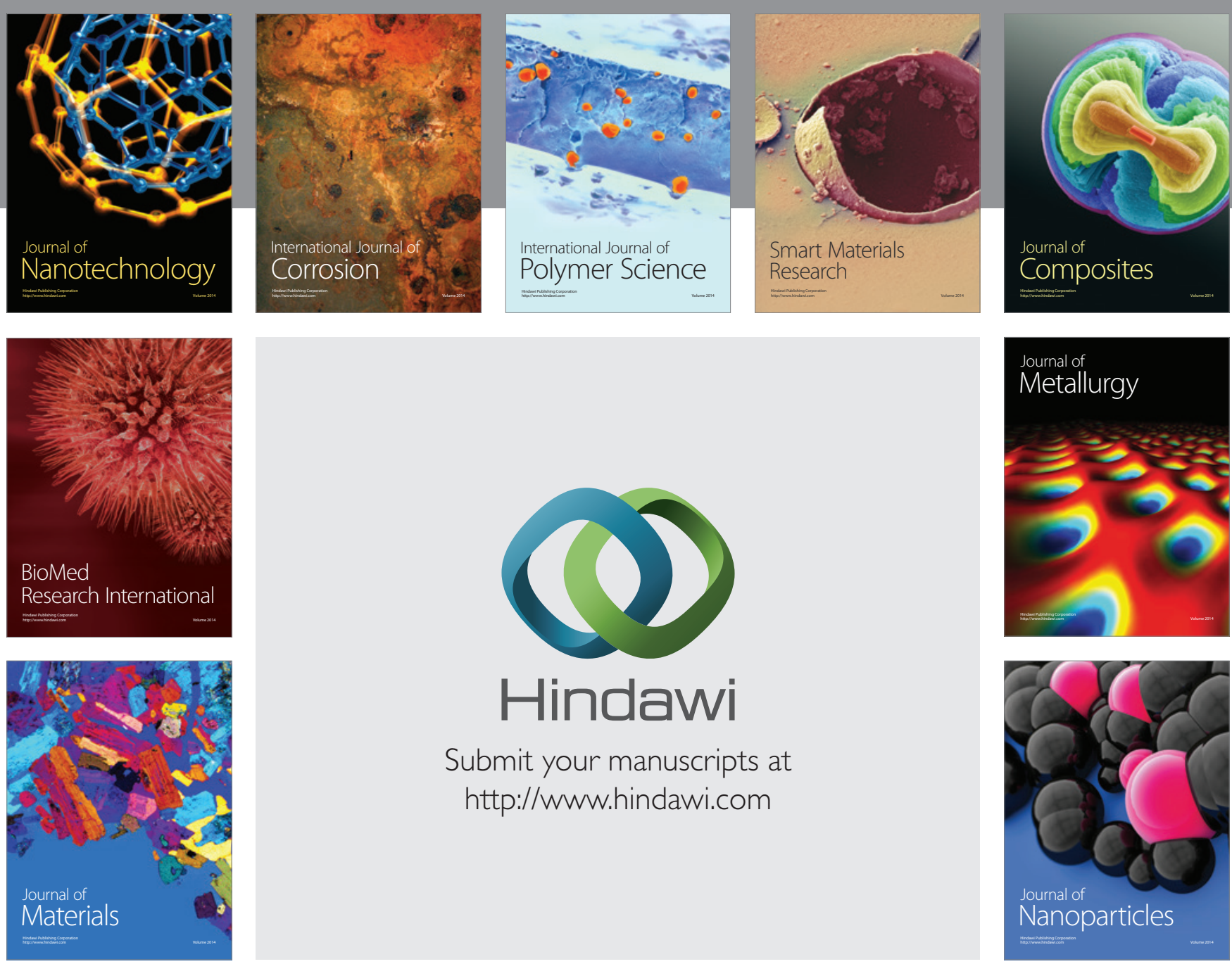

Submit your manuscripts at http://www.hindawi.com
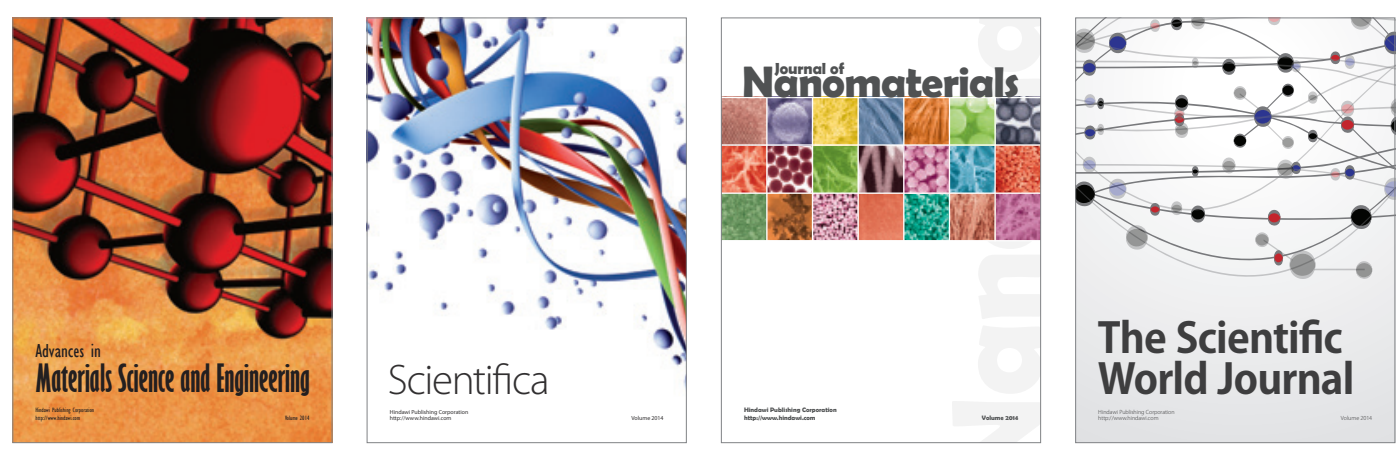

\section{The Scientific World Journal}
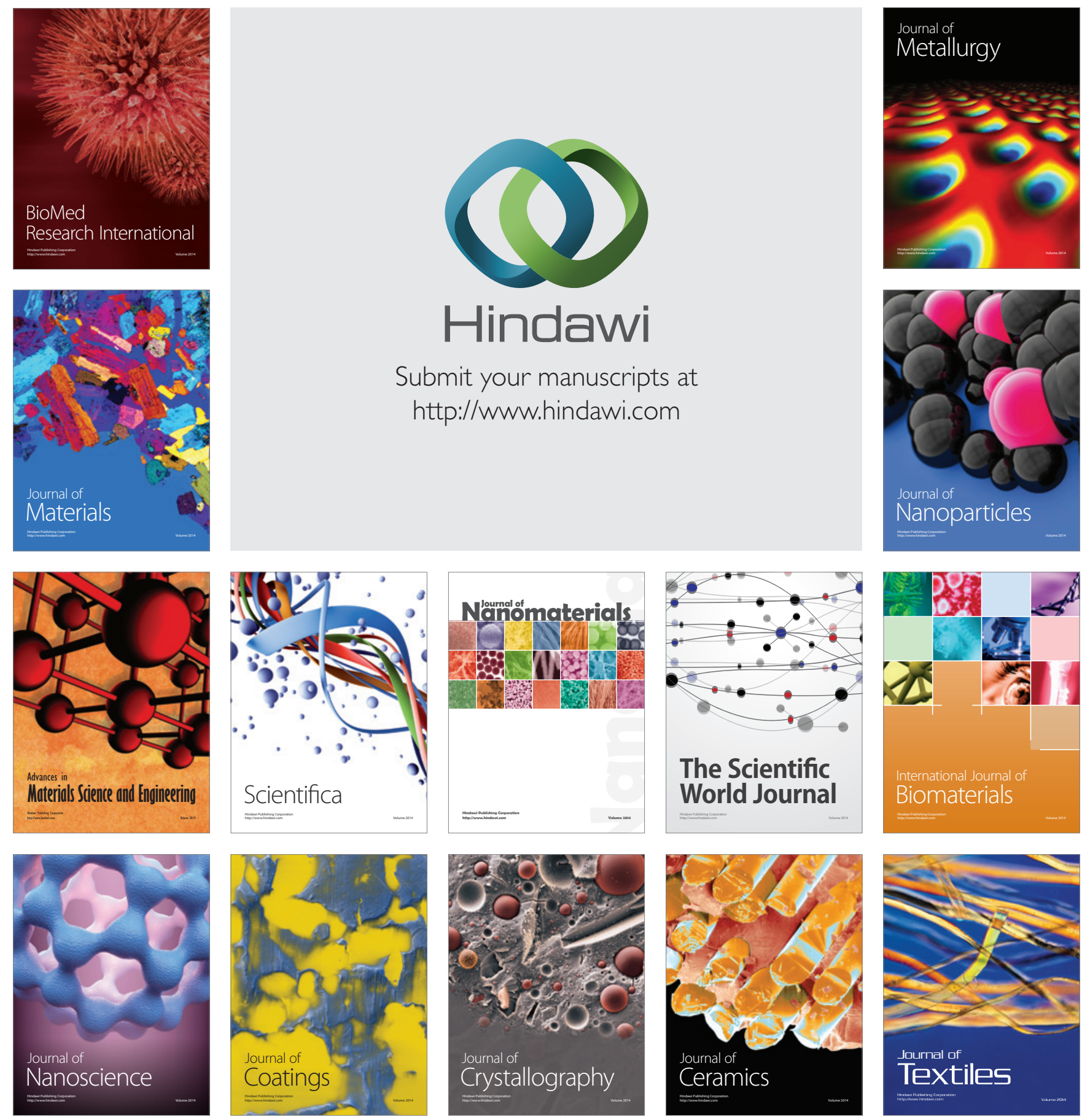